\title{
Long-term millimeter VLBI monitoring of M 87 with KVN at milliarcsecond resolution: nuclear spectrum
}

\author{
Jae-Young Kim ${ }^{1}$, Sang-Sung Lee ${ }^{2,3}$, Jeffrey A. Hodgson ${ }^{2}$, Juan-Carlos Algaba ${ }^{2,4}$, Guang-Yao Zhao ${ }^{2}$, \\ Motoki Kino ${ }^{2}$, Do-Young Byun ${ }^{2}$, and Sincheol Kang ${ }^{2,3}$
}

\author{
${ }^{1}$ Max-Planck-Institut für Radioastronomie, Auf dem Hügel 69, 53121 Bonn, Germany \\ e-mail: jykim@mpifr-bonn.mpg.de \\ ${ }^{2}$ Korea Astronomy and Space Science Institute, 776 Daedeokdae-ro, Yuseong-gu, Daejeon 30455, Korea \\ ${ }^{3}$ Korea University of Science and Technology, 217 Gajeong-ro, Yuseong-gu, Daejeon 34113, Korea \\ ${ }^{4}$ Department of Physics and Astronomy, Seoul National University, 1 Gwanak-ro, Gwanak-gu, Seoul 08826, Korea
}

Received 5 December 2017 / Accepted 25 January 2018

\begin{abstract}
We study the centimeter- to millimeter-wavelength synchrotron spectrum of the core of the radio galaxy M 87 at $\lesssim 0.8$ mas $\sim 110 R_{\mathrm{S}}$ spatial scales using four years of fully simultaneous, multi-frequency VLBI data obtained by the Korean VLBI Network (KVN). We find a core spectral index $\alpha$ of $\gtrsim-0.37\left(S \propto v^{+\alpha}\right)$ between 22 and $129 \mathrm{GHz}$. By combining resolution-matched flux measurements from the Very Long Baseline Array (VLBA) at $15 \mathrm{GHz}$ and taking the Event Horizon Telescope (EHT) $230 \mathrm{GHz}$ core flux measurements in epochs 2009 and 2012 as lower limits, we find evidence of a nearly flat core spectrum across 15 and $129 \mathrm{GHz}$, which could naturally connect the $230 \mathrm{GHz}$ VLBI core flux. The extremely flat spectrum is a strong indication that the jet base does not consist of a simple homogeneous plasma, but of inhomogeneous multi-energy components, with at least one component with the turn-over frequency $\gtrsim 100 \mathrm{GHz}$. The spectral shape can be qualitatively explained if both the strongly (compact, optically thick at $>100 \mathrm{GHz}$ ) and the relatively weakly magnetized (more extended, optically thin at $<100 \mathrm{GHz}$ ) plasma components are colocated in the footprint of the relativistic jet.
\end{abstract}

Key words. galaxies: active - galaxies: individual: M 87 - techniques: interferometric

\section{Introduction}

Located at 16.7 Mpc (Bird et al. 2010) from the Milky Way, M 87 is one of the nearest radio galaxies that contains an extraordinary massive central black hole $M_{\mathrm{BH}} \approx 6.1 \times 10^{9} M_{\odot}$ (Gebhardt et al. 2011). Some studies suggest a twice lower value of $M_{\mathrm{BH}}$ (e.g., Walsh et al. 2013). In order to be consistent with other VLBI studies of $\mathrm{M} 87$, we adopt $M_{\mathrm{BH}}=6.1 \times 10^{9} M_{\odot}$. The close distance and the large $M_{\mathrm{BH}}$ provides an excellent spatial resolution ( 1 milliarcsecond $\sim 0.08 \mathrm{pc}$ or $\sim 140$ Schwarzschild radii $\left.\left(R_{\mathrm{S}}\right)\right)$. This makes M 87 a more than suitable target for observing and studying physical conditions in the relativistic jet launching, collimation, and acceleration region by very long baseline interferometry (VLBI) observations (Kovalev et al. 2007; Ly et al. 2007; Hada et al. 2011, 2016; Asada \& Nakamura 2012; Doeleman et al. 2012; Mertens et al. 2016; Kim et al. 2016; Walker et al. 2016).

The electron energy distribution and the magnetic field strength near the central engine are critical elements in relativistic jet launching models (e.g., Blandford \& Znajek 1977; Blandford \& Payne 1982). In particular, a stronger magnetic field helps effective jet formation (see Yuan \& Narayan 2014 for a review). These models prefer a significantly inverted or at least flat synchrotron spectrum up to $>100 \mathrm{GHz}$ (Broderick \& Loeb 2009; Kino et al. 2015; Punsly 2017). A high turn-over frequency like this would imply a strong magnetic field strength $B \sim 100 \mathrm{G}$ in the jet base (Kino et al. 2015). However, the previous studies of M 87 are based on data from vastly different observing times and spatial scales.
For this reason, simultaneous multi-frequency VLBI observations of M87 in the millimeter regime can provide better observational constraints for the theoretical models. However, such an observation has been challenging. One of the main difficulties has been the limited frequency coverage offered by most VLBI observatories ( $\leq 86 \mathrm{GHz}=3.5 \mathrm{~mm}$; Hada et al. 2016; Kim et al. 2016). Recent Event Horizon Telescope (EHT) observations studied M 87 at $230 \mathrm{GHz}(1.3 \mathrm{~mm}$; e.g., Doeleman et al. 2012), but the large frequency gap between 86 and $230 \mathrm{GHz}$ and technical difficulties for submillimeter VLBI remain challenging. Furthermore, the VLBI core of M 87 varies in flux on timescales of at least weeks, especially during the ejection of a new VLBI feature from the core region (e.g., Acciari et al. 2009; Hada et al. 2014). The long-term variability properties in this frequency range are also poorly known.

In this Letter, we present a study of the M 87 VLBI core spectrum based on the fully simultaneously measured core flux at $22,43,86 \mathrm{GHz}$ and up to $129 \mathrm{GHz}$ by the Korean VLBI Network (KVN) over the past four years. We especially investigate the core spectrum at short millimeter-wavelengths and attempt to clarify whether the nuclear region has a significantly inverted or steep spectrum.

\section{Observations and data processing}

M 87 was monitored regularly by the KVN between Dec. 2012 and Dec. 2016 (31 epochs) as one of the samples included in the interferometric monitoring of gamma-ray bright AGN 


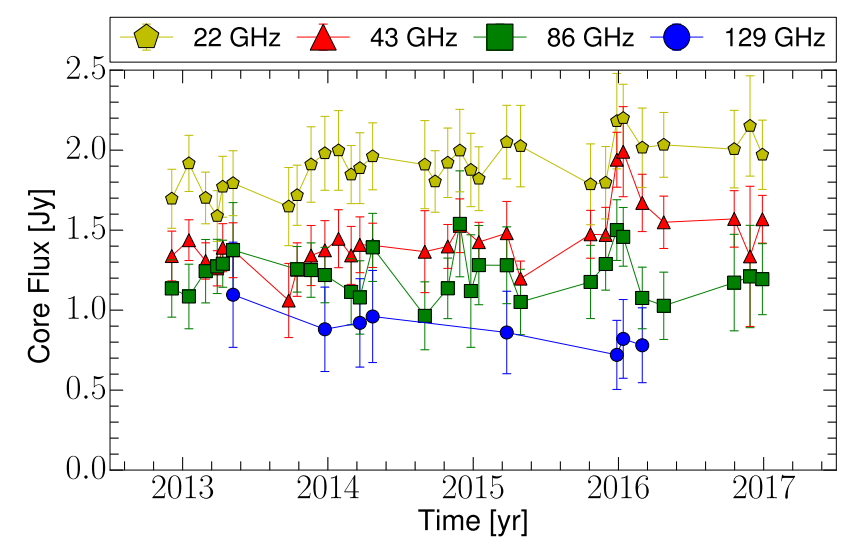

Fig. 1. Four-frequency core flux light curves obtained by the KVN observations.

(iMOGABA) program (e.g., Lee et al. 2016) ${ }^{1}$. The observations were performed simultaneously at four observing frequencies $v$ of $21.700-21.764 \mathrm{GHz}$ ( $K$ band), 43.400-43.464 GHz ( $Q$ band), 86.800-86.864 GHz ( $W$ band), and 129.300-129.364 GHz ( $D$ band). Each band has $64 \mathrm{MHz}$ of total bandwidth. The source was observed in left-circular polarization. Details on the iMOGABA program (data acquisition, correlation, postprocessing, and calibration, especially concerning the frequencyphase transfer) are provided elsewhere (see Lee et al. 2015, 2016; Algaba et al. 2015; Hodgson et al. 2016). Owing to the frequency-phase transfer technique, we detected fringes at the higher frequency bands (e.g., 86 and $129 \mathrm{GHz}$ ). After detection of the fringes, we averaged the calibrated data in $30 \mathrm{~s}$ intervals at $K$ and $Q$ band, and in $10 \mathrm{~s}$ intervals at $W$ and $D$ band to avoid amplitude loss from decoherence. Occasional station or receiver problems and poor weather conditions led to unreliable amplitude and large residual phase errors in a certain portion of the data, especially at $D$ band. We excluded these data in the further analysis.

We used the DIFMAP package (Shepherd et al. 1994) for imaging using CLEAN and phase self-calibration loops, applying natural weighting (no amplitude self-calibration because there are only three KVN stations). An asymmetric and extended jet structure was revealed at 22 and $43 \mathrm{GHz}$ by excess of fluxes in the dirty map and also by non-zero closure phases. However, the 86 and $129 \mathrm{GHz}$ data did not show a clear signature of extended jet emission (see Fig. 2 in Lee et al. 2016 for typical visibility distributions and source images). We fitted a circular Gaussian to the self-calibrated visibilities using the MODELFIT procedure in DIFMAP to estimate the total flux density $S_{\text {tot }}$ (in Jy), the FWHM size $d$ (in mas), and the peak flux density $S_{\text {peak }}$ (in mJy beam ${ }^{-1}$ ) of the core. We estimated uncertainties in the model-fit parameters and practical resolution limit of the array by following Lee et al. (2016), accounting for the effect of the finite signal-to-noise ratio $(\mathrm{S} / \mathrm{N})$ that varies from session to session. At $D$ band, the systematic flux uncertainty can be as large as 30\% (Lee et al. 2016) because of large residual phase errors. Therefore, we take the $30 \%$ as a conservative flux uncertainty at $129 \mathrm{GHz}$. We also investigated possible amplitude loss at $D$ band due to the decoherence by averaging the data over different timescales (from 2 to $60 \mathrm{~s}$ ) and measuring the peak intensity in the clean image. We found that the $10 \mathrm{~s}$ averaging can cause $\lesssim 10 \%$ of amplitude loss. This is not significant compared to the $30 \%$ flux uncertainty. Thus, we ignore the decoherence in the following discussions.

\footnotetext{
1 http://radio.kasi.re.kr/sslee/
}

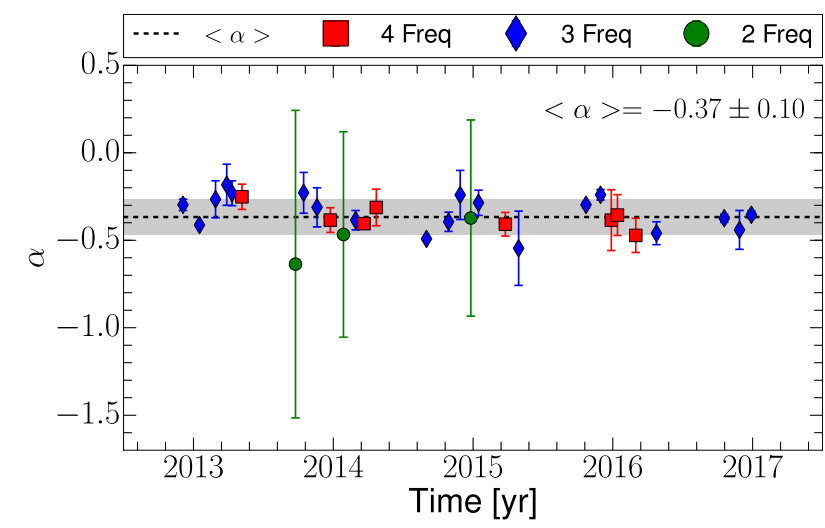

Fig. 2. Spectral index light curve $\left(S \propto v^{+\alpha}\right)$. Different symbols in each epoch denote the different number of frequencies available for the power-law fit. The broken line and the shaded region denote the mean and standard deviation of $\alpha$, respectively.

\section{Results and analysis}

\subsection{Core model-fit properties}

In Table B.1 we show a summary of the observations and the results of the core flux measurements. We find that $45 \%, 65 \%$, $76 \%$, and $38 \%$ of all the model components are spatially resolved at $K, Q, W$, and $D$ band, respectively. The mean FWHM sizes of the resolved components are $0.8 \pm 0.3,0.55 \pm 0.11,0.42 \pm$ 0.13 , and $0.23 \pm 0.09$ mas at $K, Q, W$, and $D$ bands, where the uncertainties correspond to the standard deviation of each distributions. Higher resolution VLBI observations show that the source consists of a more compact core and an extended, complex jet (Kovalev et al. 2007; Ly et al. 2007; Hada et al. 2016; Kim et al. 2016). Therefore, the KVN multi-frequency observations probe synchrotron emission from the mixed structure on scales smaller than $\sim 0.8$ mas (equivalently $\sim 110 R_{\mathrm{s}}$ projected linear size).

Our multi-frequency light curves are shown in Fig. 1. The core does not show significant flux variability over $1 \sigma$ during most of the observing epochs. However, we note that the core flux increased from $\sim 1.5$ to $\sim 2.0 \mathrm{Jy}$ at $43 \mathrm{GHz}$ and similarly at 22 and $86 \mathrm{GHz}$ in the beginning of 2016. A study of this flux enhancement will be presented in a forthcoming publication. The time-averaged core flux (including both resolved and unresolved components) are $1.90 \pm 0.15,1.44 \pm 0.19,1.22 \pm 0.14$, and $0.88 \pm$ $0.11 \mathrm{Jy}$ at $K, Q, W$, and $D$ band, respectively. These values are broadly consistent with previously reported flux values of the M 87 nuclear region on a similar spatial scale. For instance, the $\mathrm{KVN} Q$-band flux $(\sim 1.4 \mathrm{Jy})$ is comparable to the M 87 core flux measured within 1.2 mas from the intensity peak by the Very Long Baseline Array (VLBA) $43 \mathrm{GHz}$ observations ( 1.2 Jy; Acciari et al. 2009).

\subsection{Synchrotron spectrum analysis}

Based on the absence of a significant synchrotron peak between $22-129 \mathrm{GHz}$ and given a total of four frequencies available, we modeled the core spectrum by a single power-law, that is, $S_{\text {tot }} \propto v^{+\alpha}$, where $\alpha$ is the optically thin spectral index (negative for optically thin plasma). In each epoch, we used the core flux from as many available frequencies as possible. In Fig. 2 we show the spectral index light curve and in Fig. 3 the core flux versus the frequency. The spectral index shows some oscillatory pattern with a $\sim 1$ yr timescale and a weak 


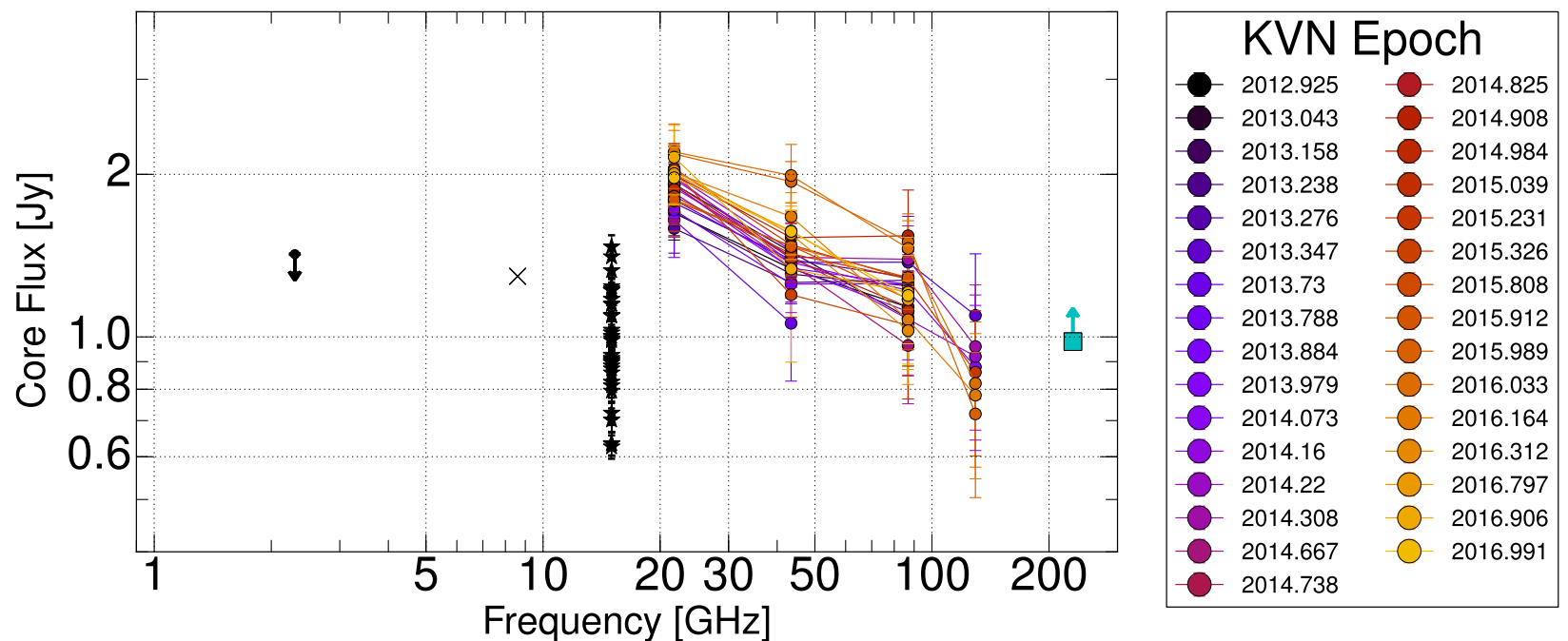

Fig. 3. Radio spectrum of the M 87 core region in log-log scale. Different KVN observing epochs are indicated by different colors in the legend. The arrows indicate upper and lower limits. References of other multi-frequency flux information (in order of increasing radio frequency): Pushkarev \& Kovalev (2012); Lister et al. (2016); Doeleman et al. (2012); Akiyama et al. (2015).

trend of spectral steepening over a longer period. By averaging the spectral index light curve, we obtained the mean spectral index $\langle\alpha\rangle=-0.37 \pm 0.10$, which corresponds to a mildly steep spectrum.

However, we note that the limited $(u, v)$ coverage of KVN could have a significant effect on the core spectral index measurement because of the different array sensitivity to extended emission at different frequencies. In order to study the effect of the $(u, v)$ coverage, we have performed a dedicated imaging simulation (see Appendix A). We find that an artificial spectral steepening of $\Delta \alpha \sim-0.2$ could be possible for the M 87-like jet structure only due to the $(u, v)$-coverage effect (equivalent to $\sim 36 \%$ of flux decrease from 22 to $129 \mathrm{GHz}$ ). Furthermore, M 87 has a substantially extended jet, whose spectrum is quite steep already at $\gtrsim 0.2$ mas core separation ( $\alpha \lesssim-0.7$; Hovatta et al. 2014; Hada et al. 2016). Therefore, the structure blending effect could make the KVN M 87 core spectrum steeper even if the intrinsic spectrum is nearly flat. Considering the imaging simulation results, we conclude that the true spectrum of the jet base region is substantially flat up to $129 \mathrm{GHz}$.

We verified this conclusion by comparing non-simultaneous M 87 core flux information that is available from earlier publications. Here we specifically paid attention to the different resolutions of different observations in order to compare the fluxes on a comparable angular resolution. First, Pushkarev \& Kovalev (2012) reported an $8.6 \mathrm{GHz}$ core flux of $\sim 1.3 \mathrm{Jy}$ and an FWHM size of $\sim 0.6$ mas. The authors also reported a $2.3 \mathrm{GHz}$ core flux of $\sim 1.4 \mathrm{Jy}$ and an FWHM size of $\sim 2.6$ mas along the major axis of an elliptical Gaussian. Second, we made use of the M 87 core information provided by the MOJAVE program (Lister et al. 2016; 34 epochs of VLBA $15 \mathrm{GHz}$ ). The time-averaged core flux and the FWHM size are $0.99 \pm 0.22 \mathrm{Jy}$ and $0.26 \pm 0.04$ mas, respectively. Finally, we refer to the $230 \mathrm{GHz}$ EHT observations in epochs 2009 (Doeleman et al. 2012) and 2012 (Akiyama et al. 2015). At this frequency, an ultra-compact plasma ( 40 $\mu$ as) has a flux of $\sim 1 \mathrm{Jy}$. We take the $2.3 \mathrm{GHz}$ flux as the upper limit since the $2.3 \mathrm{GHz}$ size is an order-of-magnitude larger with regard to the KVN results. For similar reasons, we regard the EHT flux as the lower limit at $230 \mathrm{GHz}$.

We find that the global radio spectrum of the core is considerably flat without a clear signature of inverted or steep spectra
(Fig. 3). In particular, the long-term $15 \mathrm{GHz}$ core flux is quite comparable to our four-year measurements made by the KVN at 86 and $129 \mathrm{GHz}$. Since the angular resolution of the KVN at short millimeter wavelengths is comparable to that of VLBA at $15 \mathrm{GHz}$, we can calculate a non-simultaneous but resolutionmatched spectral index, finding $\alpha_{(15-129) \mathrm{GHz}}=-0.03 \pm 0.23$ between 15-129 GHz. Such a flat spectrum agrees with the $230 \mathrm{GHz}$ flux lower limit constrained by the EHT observations. Although the $22 \mathrm{GHz} \mathrm{KVN}$ flux appears slightly high compared to the $15 \mathrm{GHz}$ flux, we note that this is most likely due to the blending effect we mentioned in Sect. 3.1. The resolution-matched core flux between $5-22 \mathrm{GHz}$ indeed shows a substantially flat spectrum within $\sim 1$ mas from the intensity peak (see Fig. 4 of Hada et al. 2012). Therefore, we conclude that the M 87 core on a submilliarcsecond scale has a substantially flat radio spectrum between $15-129 \mathrm{GHz}$ and possibly up to $230 \mathrm{GHz}$.

\section{Discussion}

\subsection{Comparison with theoretical predictions}

It has been routinely considered that featureless flat spectra of extragalactic jet sources are made by inhomogeneous plasma consisting of multiple components with different turn-over frequencies (i.e., "cosmic conspiracy"; Cotton et al. 1980). Our KVN data do not allow for a reliable spectral decomposition because they lack spatial resolution. However, the flat spectrum up to at least $129 \mathrm{GHz}$ suggests the coexistence of qualitatively different plasma with low $(<100 \mathrm{GHz})$ and high synchrotron peak frequencies $(>100 \mathrm{GHz})$. We can interpret the latter based on theoretical studies of energetics of relativistic plasma near the central engine of M 87. This connection is provided by the fact that synchrotron radiation from the core region of M 87 at short millimeter wavelengths originates from the vicinity of its central BH (Hada et al. 2011). In this regard, a peak frequency higher than $100 \mathrm{GHz}$ supports the broadly investigated idea about a jet dominated by magnetic energy that is launched at the base of the M 87 jet (Broderick \& Loeb 2009; Kino et al. 2015; Punsly 2017).

The underlying flat spectrum in the intermediate frequency range $(\sim 15-86 \mathrm{GHz})$ is also interesting. This cannot be easily 
explained by the expanding jet because the jet has a turn-over frequency below $24 \mathrm{GHz}$ already at a core distance of $\gtrsim 0.2$ mas (Hada et al. 2016). Interestingly, Kino et al. (2015) showed that a single, homogeneous, ultra-compact ( $\sim 40 \mu$ as), and fully synchrotron self-absorbed (SSA) plasma at $230 \mathrm{GHz}$ would cause problems for several reasons. The authors specifically demonstrated that the compact size and the high turn-over frequency imply a magnetic field strength of $B \approx 300 \mathrm{G}$. This value leads to an unrealistically great electromagnetic jet power and extremely short synchrotron cooling timescale, which contradicts observations (e.g., Doeleman et al. 2012). Based on this, the authors suggested that the jet base region consists of at least two zones with different levels of magnetization, which could produce another synchrotron peak in the intermediate-frequency regime.

\subsection{Constraining the turn-over frequency of the magnetic energy dominated plasma}

Because we lack direct flux measurements between 129 and $230 \mathrm{GHz}$, it is unclear whether the highest turn-over frequency can be even higher than $230 \mathrm{GHz}$ or if the entire jet base becomes optically thin at $230 \mathrm{GHz}$. In the latter case, Kino et al. (2015) suggested the spectrum turn-over to be at $160 \mathrm{GHz}$ (see Fig. 5 therein) and a very steep spectrum $(\alpha \leq-2.5)$ between 160 and $230 \mathrm{GHz}$. In order to examine the latter case, we took the $129 \mathrm{GHz}$ core flux of $\sim 0.9 \mathrm{Jy}$ from the KVN data as an upper limit for the $129 \mathrm{GHz}$ flux on the EHT-scale region $(\lesssim 40 \mu \mathrm{as})$. We also assumed a flat spectrum between 129 and $160 \mathrm{GHz}$, but a steep spectrum between 160 and $230 \mathrm{GHz}$. This gives the $230 \mathrm{GHz}$ flux upper limit at $230 \mathrm{GHz}$ of $0.9 \mathrm{Jy} \times(230 / 160)^{-2.5} \sim$ $0.4 \mathrm{Jy}$. Even a much more moderate steep spectral index $\alpha=$ -0.7 predicts $0.9 \mathrm{Jy} \times(230 / 160)^{-0.7} \sim 0.7 \mathrm{Jy}$ at $230 \mathrm{GHz}$. These fluxes are not sufficient to explain the $230 \mathrm{GHz}$ flux measured by the EHT observations. Therefore, we favor the former scenario.

\section{Summary}

In this Letter, we have presented a study of the synchrotron spectrum of the nuclear region in M 87 by KVN VLBI monitoring observations that have been regularly performed over four years at 22, 43, 86, and $129 \mathrm{GHz}$. The KVN observations resolved the core at a projected linear size of $\lesssim 0.8$ mas $\sim 110 R_{\mathrm{s}}$. The KVN data constrained the core spectral index $\alpha \gtrsim-0.37$ between 22-129 GHz. Other resolution-matched multi-frequency observations suggest the flat spectrum over a wider range of frequency $(15-230 \mathrm{GHz})$. The flat spectrum extending up to short millimeter wavelengths implies a strong magnetization in the jet base, consistent with the theoretical predictions (e.g., Broderick \& Loeb 2009; Kino et al. 2015). Therefore, we suggest a magnetically dominated jet-launching scenario for M 87.
Acknowledgements. We thank the anonymous referee for the careful reading and valuable comments that helped us to improve the paper. J.-Y. Kim is supported for this research by the International Max Planck Research School (IMPRS) for Astronomy and Astrophysics at the University of Bonn and Cologne. J.-Y. Kim would like to thank Eduardo Ros, Manel Perucho, and Christian Fromm for fruitful discussions. S.-S. Lee was supported by the National Research Foundation of Korea (NRF) grant funded by the Korea government (MSIP No. NRF-2016R1C1B2006697). G.-Y. Zhao is supported by the Korea Research Fellowship Program through the National Research Foundation of Korea (NRF) funded by the Ministry of Science and ICT (NRF-2015H1D3A1066561). We are grateful to all staff members in KVN who helped to operate the array and to correlate the data. The KVN is a facility operated by KASI (Korea Astronomy and Space Science Institute). The KVN operations are supported by KREONET (Korea Research Environment Open NETwork), which is managed and operated by KISTI (Korea Institute of Science and Technology Information). This research has made use of data from the MOJAVE database that is maintained by the MOJAVE team (Lister et al. 2009).

\section{References}

Acciari, V. A., Aliu, E., Arlen, T., et al. 2009, Science, 325, 444 Akiyama, K., Lu, R.-S., Fish, V. L., et al. 2015, ApJ, 807, 150

Algaba, J.-C., Zhao, G.-Y., Lee, S.-S., et al. 2015, J. Korean Astron. Soc., 48, 237

Asada, K., \& Nakamura, M. 2012, ApJ, 745, L28

Bird, S., Harris, W. E., Blakeslee, J. P., \& Flynn, C. 2010, A\&A, 524, A71

Blandford, R. D., \& Payne, D. G. 1982, MNRAS, 199, 883

Blandford, R. D., \& Znajek, R. L. 1977, MNRAS, 179, 433

Broderick, A. E., \& Loeb, A. 2009, ApJ, 697, 1164

Cotton, W. D., Wittels, J. J., Shapiro, I. I., et al. 1980, ApJ, 238, L123

Doeleman, S. S., Fish, V. L., Schenck, D. E., et al. 2012, Science, 338, 355

Fromm, C. M., Ros, E., Perucho, M., et al. 2013, A\&A, 557, A105

Gebhardt, K., Adams, J., Richstone, D., et al. 2011, ApJ, 729, 119

Greisen, E. W. 1990, in Acquisition, Processing and Archiving of Astronomical Images, eds. G. Longo, \& G. Sedmak, 125

Hada, K., Doi, A., Kino, M., et al. 2011, Nature, 477, 185

Hada, K., Kino, M., Nagai, H., et al. 2012, ApJ, 760, 52

Hada, K., Giroletti, M., Kino, M., et al. 2014, ApJ, 788, 165

Hada, K., Kino, M., Doi, A., et al. 2016, ApJ, 817, 131

Hodgson, J. A., Lee, S.-S., Zhao, G.-Y., et al. 2016, J. Korean Astron. Soc., 49, 137

Hovatta, T., Aller, M. F., Aller, H. D., et al. 2014, AJ, 147, 143

Kim, J.-Y., Lu, R.-S., Krichbaum, T., et al. 2016, Galaxies, 4, 39

Kino, M., Takahara, F., Hada, K., et al. 2015, ApJ, 803, 30

Kovalev, Y. Y., Lister, M. L., Homan, D. C., \& Kellermann, K. I. 2007, ApJ, 668 L27

Lee, S.-S., Oh, C. S., Roh, D.-G., et al. 2015, J. Korean Astron. Soc., 48, 125

Lee, S.-S., Wajima, K., Algaba, J.-C., et al. 2016, ApJS, 227, 8

Lister, M. L., Aller, H. D., Aller, M. F., et al. 2009, AJ, 137, 3718

Lister, M. L., Aller, M. F., Aller, H. D., et al. 2016, AJ, 152, 12

Ly, C., Walker, R. C., \& Junor, W. 2007, ApJ, 660, 200

Mertens, F., Lobanov, A. P., Walker, R. C., \& Hardee, P. E. 2016, A\&A, 595, A54

Punsly, B. 2017, ApJ, 850, 190

Pushkarev, A. B., \& Kovalev, Y. Y. 2012, A\&A, 544, A34

Shepherd, M. C., Pearson, T. J., \& Taylor, G. B. 1994, BAAS, 26, 987

Walker, R. C., Hardee, P. E., Davies, F., et al. 2016, Galaxies, 4, 46

Walsh, J. L., Barth, A. J., Ho, L. C., \& Sarzi, M. 2013, ApJ, 770, 86

Yuan, F., \& Narayan, R. 2014, ARA\&A, 52, 529 


\section{Appendix A: Simulation of KVN visibilities and estimation of a systematic trend in the spectral index}

The analysis of multi-frequency interferometric images is sensitive to the finite sampling of the source information in the visibility domain (e.g., Fromm et al. 2013). This issue can be particularly important for the KVN data because of the limited $(u, v)$ coverage of the array (see Fig. 2 of Lee et al. 2016). Therefore, we evaluated the effect of the frequency-dependent $(u, v)$ coverage on the accuracy of the spectral index measurement.

The main goal was to feed the simulator with exactly the same source model with a flat spectrum for different $(u, v)$ coverages (i.e., only different observing frequencies) and test if an absolutely flat spectral index can be recovered from the simulated data.

For this purpose, we prepared a ground-truth image of the M 87 jet to simulate synthetic KVN observations. A full-track M 87 VLBA $15 \mathrm{GHz}$ data set was taken from the MOJAVE database (VLBA code BK145A), and we imaged the source structure using the DIFMAP task CLEAN. The maximum baseline length of this observation was $440 \mathrm{M} \lambda$ and provided an angular resolution of $0.45 \times 0.84$ mas. In panel (a) of Fig. A.1 we show the corresponding inner jet structure. This clean model was imported into the NRAO AIPS software (Greisen 1990) to be used as an input for the AIPS task UVCON. Then, we generated synthetic datasets using exactly the same input clean model for the four different $\mathrm{KVN}$ observing frequencies. The entire simulation was performed by following several specific steps.

1. A full-track synthetic data set was created by using AIPS UVCON. We assumed one of the KVN observing frequencies (e.g., $22 \mathrm{GHz}$ ), the KVN station coordinates, and a $10 \mathrm{~h}$ observing run. System temperatures and aperture efficiencies were chosen to be close to typical values in real observations. We note that the decoherence was not considered in the simulation.

2. From session to session, KVN observed M87 in snapshot mode at different baseline position angles. In order to account for this, we split the simulated full-track data into five subsets, each of which had the same $2 \mathrm{~h}$ of onsource time (e.g., LST 6:00-8:00, 8:00-10:00, 10:00-12:00, 12:00-14:00, and 14:00-16:00).

3. Each sub-dataset was loaded into DIFMAP, and we estimated the core flux by fitting a circular Gaussian model to the visibilities, as described in Sect. 2 (see panel (b) of Fig. A.1).

4. The core flux estimate was repeated for all the five subdatasets. Then, we took the average and standard deviation of the five different flux values to determine a characteristic flux value.

5. Steps 1 to 4 were repeated for the synthetic data sets at observing frequencies of $22,43,86$, and $129 \mathrm{GHz}$.

In Fig. A. 2 we show the artificial spectrum obtained by the simulation. We were not able to recover a completely flat spectral index and rather obtained $\alpha_{\text {sim }}$ of $-0.23 \pm 0.02$. Most likely, this is due to different level of the array sensitivity to extended emission at limited $u v$ spacings. Therefore, the limited $(u, v)$ coverage very likely causes similar systematic effects in the analysis of the real multi-frequency M 87 data as well.

After these considerations, we conclude that it is unlikely that the actual source spectrum is even slightly steep or inverted in our observing band.
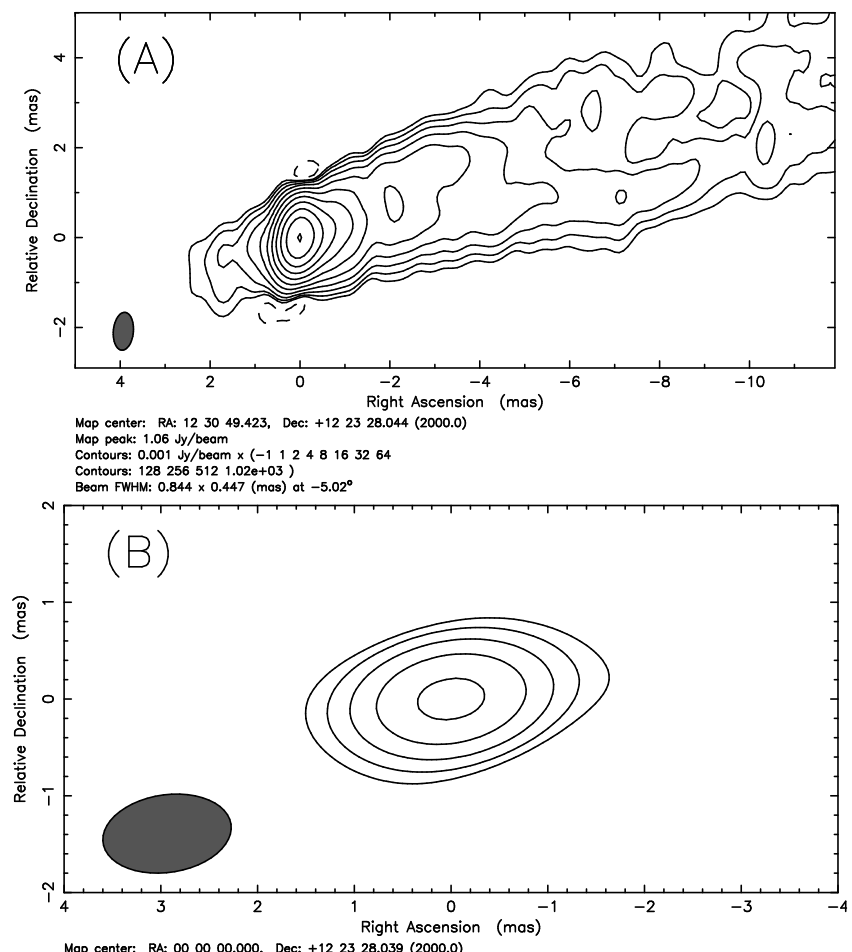

Mop center: RA: 000000.000 , Dec: $+122328.039(2000.0)$

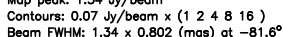

Fig. A.1. Panel $a$ : input M 87 clean model image obtained by the VLBA observations at $15 \mathrm{GHz}$. Panel $b$ : KVN $86 \mathrm{GHz}$ image obtained from a simulated $2 \mathrm{~h}$ long KVN snapshot observation of the model image in panel a.

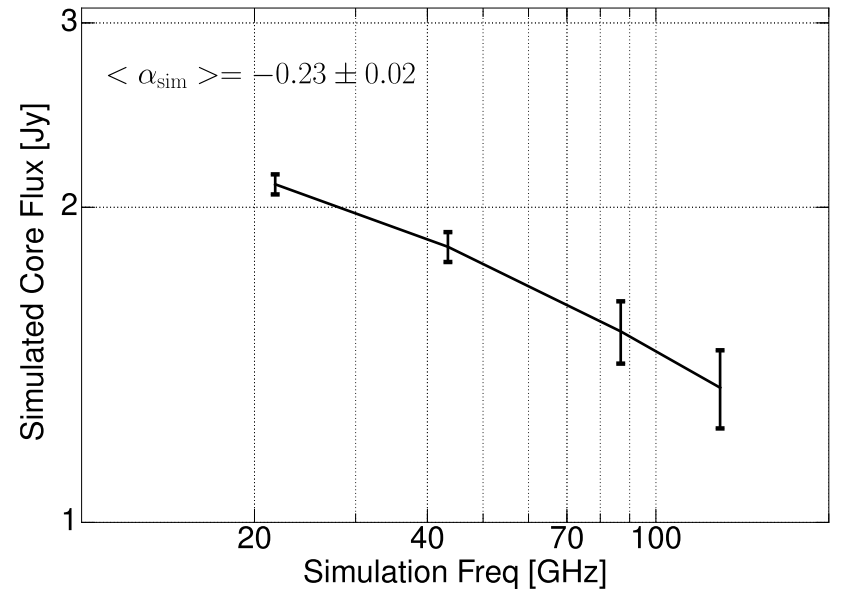

Fig. A.2. Artificial M 87 core flux versus the simulation frequency obtained by the simulated observations. 
Appendix B: Observing epochs and M 87 core model-fit parameters

Table B.1. Observing epochs and M 87 core model-fit parameters.

\begin{tabular}{|c|c|c|c|c|c|c|c|c|c|}
\hline $\begin{array}{c}\text { Epoch } \\
\text { (yyyy-mm-dd) } \\
(1)\end{array}$ & MJD & $\begin{array}{c}v_{\mathrm{obs}} \\
(\mathrm{GHz}) \\
(3) \\
\end{array}$ & $\begin{array}{c}S_{v} \\
(\mathrm{Jy}) \\
(4)\end{array}$ & $\begin{array}{c}S_{\text {peak }} \\
\left(\mathrm{Jy} \mathrm{beam}^{-1}\right) \\
(5)\end{array}$ & $\begin{array}{c}d \\
(\mathrm{mas}) \\
(6) \\
\end{array}$ & $\begin{array}{c}\sigma_{\mathrm{rms}} \\
\left(\mathrm{mJy} \mathrm{beam}^{-1}\right) \\
(7)\end{array}$ & $\begin{array}{c}B_{\mathrm{maj}} \\
(\mathrm{mas}) \\
(8) \\
\end{array}$ & $\begin{array}{c}B_{\min } \\
(\mathrm{mas}) \\
(9) \\
\end{array}$ & $\begin{array}{c}B_{\mathrm{PA}} \\
(\mathrm{deg}) \\
(10)\end{array}$ \\
\hline \multirow[t]{3}{*}{ 2012-12-04 } & 56265 & 21.7 & $1.7 \pm 0.2$ & $1.7 \pm 0.1$ & $<0.31$ & 10.0 & 5.70 & 3.22 & -68.0 \\
\hline & & 43.4 & $1.3 \pm 0.2$ & $1.3 \pm 0.1$ & $<0.17$ & 9.0 & 2.84 & 1.61 & -66.3 \\
\hline & & 86.8 & $1.1 \pm 0.2$ & $1.1 \pm 0.1$ & $0.18 \pm 0.02$ & 13.9 & 1.39 & 0.83 & -67.0 \\
\hline \multirow[t]{3}{*}{ 2013-01-16 } & 56308 & 21.7 & $1.9 \pm 0.2$ & $1.8 \pm 0.1$ & $0.91 \pm 0.06$ & 8.0 & 5.33 & 3.32 & -79.5 \\
\hline & & 43.4 & $1.4 \pm 0.1$ & $1.3 \pm 0.1$ & $0.54 \pm 0.04$ & 5.6 & 2.67 & 1.66 & -81.0 \\
\hline & & 86.8 & $1.1 \pm 0.2$ & $0.8 \pm 0.1$ & $0.53 \pm 0.08$ & 17.8 & 1.49 & 0.81 & -63.4 \\
\hline \multirow[t]{3}{*}{ 2013-02-27 } & 56350 & 21.7 & $1.7 \pm 0.2$ & $1.7 \pm 0.1$ & $<0.27$ & 7.6 & 5.43 & 3.36 & -84.9 \\
\hline & & 43.4 & $1.3 \pm 0.1$ & $1.2 \pm 0.1$ & $0.56 \pm 0.04$ & 4.9 & 2.76 & 1.64 & -89.1 \\
\hline & & 86.8 & $1.2 \pm 0.2$ & $1.0 \pm 0.1$ & $0.50 \pm 0.06$ & 15.2 & 1.43 & 0.82 & -88.5 \\
\hline \multirow[t]{3}{*}{ 2013-03-28 } & 56380 & 21.7 & $1.6 \pm 0.2$ & $1.6 \pm 0.1$ & $0.58 \pm 0.04$ & 7.9 & 5.33 & 3.26 & -78.3 \\
\hline & & 43.4 & $1.3 \pm 0.1$ & $1.2 \pm 0.1$ & $0.38 \pm 0.02$ & 4.8 & 2.66 & 1.66 & -75.9 \\
\hline & & 86.8 & $1.3 \pm 0.2$ & $1.0 \pm 0.1$ & $0.52 \pm 0.05$ & 10.9 & 1.35 & 0.79 & -80.4 \\
\hline \multirow[t]{3}{*}{ 2013-04-11 } & 56394 & 21.7 & $1.8 \pm 0.2$ & $1.8 \pm 0.1$ & $0.40 \pm 0.03$ & 10.2 & 5.41 & 3.24 & -75.3 \\
\hline & & 43.4 & $1.4 \pm 0.2$ & $1.3 \pm 0.1$ & $<0.15$ & 8.1 & 2.66 & 1.64 & -76.2 \\
\hline & & 86.8 & $1.3 \pm 0.2$ & $1.1 \pm 0.1$ & $0.52 \pm 0.05$ & 8.2 & 2.62 & 0.97 & 49.3 \\
\hline \multirow[t]{4}{*}{ 2013-05-07 } & 56419 & 21.7 & $1.8 \pm 0.2$ & $1.8 \pm 0.1$ & $0.46 \pm 0.04$ & 11.4 & 5.48 & 3.28 & -72.2 \\
\hline & & 43.4 & $1.4 \pm 0.2$ & $1.3 \pm 0.1$ & $0.50 \pm 0.05$ & 10.6 & 2.63 & 1.74 & -73.4 \\
\hline & & 86.8 & $1.4 \pm 0.3$ & $0.9 \pm 0.2$ & $0.7 \pm 0.1$ & 29.0 & 1.38 & 0.82 & -76.4 \\
\hline & & 129.3 & $1.1 \pm 0.3$ & $0.9 \pm 0.3$ & $0.4 \pm 0.1$ & 32.5 & 0.85 & 0.64 & -72.8 \\
\hline \multirow[t]{2}{*}{ 2013-09-24 } & 56559 & 21.7 & $1.7 \pm 0.2$ & $1.6 \pm 0.2$ & $0.71 \pm 0.08$ & 17.9 & 5.39 & 3.25 & -77.9 \\
\hline & & 43.4 & $1.1 \pm 0.2$ & $1.0 \pm 0.2$ & $<0.31$ & 24.7 & 2.82 & 1.52 & -78.9 \\
\hline \multirow[t]{3}{*}{ 2013-10-15 } & 56581 & 21.7 & $1.7 \pm 0.2$ & $1.7 \pm 0.1$ & $0.74 \pm 0.06$ & 10.2 & 5.34 & 3.49 & -83.3 \\
\hline & & 43.4 & $1.3 \pm 0.2$ & $1.2 \pm 0.1$ & $<0.19$ & 11.0 & 2.77 & 1.63 & -70.1 \\
\hline & & 86.8 & $1.3 \pm 0.1$ & $1.0 \pm 0.1$ & $0.57 \pm 0.05$ & 7.7 & 1.49 & 0.79 & -77.4 \\
\hline \multirow[t]{3}{*}{ 2013-11-19 } & 56616 & 21.7 & $1.9 \pm 0.2$ & $1.8 \pm 0.2$ & $<0.35$ & 14.4 & 5.19 & 3.37 & -85.0 \\
\hline & & 43.4 & $1.3 \pm 0.2$ & $1.3 \pm 0.1$ & $<0.20$ & 13.0 & 2.60 & 1.65 & -83.8 \\
\hline & & 86.8 & $1.3 \pm 0.2$ & $1.1 \pm 0.1$ & $0.38 \pm 0.04$ & 11.3 & 1.31 & 0.81 & -86.3 \\
\hline \multirow[t]{4}{*}{ 2013-12-24 } & 56650 & 21.7 & $2.0 \pm 0.2$ & $2.0 \pm 0.2$ & $<0.32$ & 13.4 & 5.28 & 3.19 & -89.5 \\
\hline & & 43.4 & $1.4 \pm 0.2$ & $1.3 \pm 0.1$ & $<0.19$ & 12.3 & 2.65 & 1.63 & -89.7 \\
\hline & & 86.8 & $1.2 \pm 0.2$ & $1.0 \pm 0.1$ & $0.39 \pm 0.04$ & 12.0 & 1.34 & 0.77 & -89.2 \\
\hline & & 129.3 & $0.9 \pm 0.3$ & $0.8 \pm 0.3$ & $<0.09$ & 15.4 & 0.98 & 0.51 & -71.4 \\
\hline \multirow[t]{2}{*}{ 2014-01-27 } & 56685 & 21.7 & $2.0 \pm 0.3$ & $1.9 \pm 0.2$ & $0.88 \pm 0.08$ & 15.4 & 5.18 & 3.35 & 89.2 \\
\hline & & 43.4 & $1.5 \pm 0.2$ & $1.4 \pm 0.1$ & $0.35 \pm 0.03$ & 11.2 & 2.57 & 1.76 & -84.6 \\
\hline \multirow[t]{3}{*}{ 2014-02-28 } & 56716 & 21.7 & $1.9 \pm 0.2$ & $1.9 \pm 0.1$ & $<0.29$ & 8.9 & 5.19 & 3.57 & -82.4 \\
\hline & & 43.4 & $1.3 \pm 0.2$ & $1.3 \pm 0.1$ & $0.51 \pm 0.05$ & 12.1 & 2.69 & 1.9 & -83.6 \\
\hline & & 86.8 & $1.1 \pm 0.2$ & $0.8 \pm 0.1$ & $0.66 \pm 0.10$ & 57.7 & 1.46 & 0.93 & 73.1 \\
\hline \multirow[t]{4}{*}{ 2014-03-22 } & 56738 & 21.7 & $1.9 \pm 0.2$ & $1.9 \pm 0.2$ & $0.66 \pm 0.06$ & 12.9 & 5.3 & 3.25 & -86.5 \\
\hline & & 43.4 & $1.4 \pm 0.2$ & $1.4 \pm 0.1$ & $0.43 \pm 0.04$ & 10.7 & 2.63 & 1.65 & -85.9 \\
\hline & & 86.8 & $1.1 \pm 0.2$ & $1.0 \pm 0.2$ & $0.22 \pm 0.03$ & 23.7 & 1.36 & 0.8 & -84.4 \\
\hline & & 129.3 & $0.9 \pm 0.3$ & $0.9 \pm 0.3$ & $<0.1$ & 18.0 & 1.0 & 0.53 & 82.0 \\
\hline \multirow[t]{4}{*}{ 2014-04-23 } & 56770 & 21.7 & $2.0 \pm 0.2$ & $1.9 \pm 0.2$ & $<0.3$ & 11.1 & 5.4 & 3.26 & -89.9 \\
\hline & & 43.4 & $1.4 \pm 0.1$ & $1.3 \pm 0.1$ & $0.43 \pm 0.03$ & 6.8 & 2.67 & 1.64 & -89.6 \\
\hline & & 86.8 & $1.4 \pm 0.2$ & $1.2 \pm 0.1$ & $0.45 \pm 0.05$ & 15.9 & 1.39 & 0.79 & -89.9 \\
\hline & & 129.3 & $1.0 \pm 0.3$ & $0.9 \pm 0.3$ & $0.15 \pm 0.04$ & 19.6 & 0.9 & 0.54 & -87.4 \\
\hline \multirow[t]{3}{*}{ 2014-09-01 } & 56902 & 21.7 & $2.0 \pm 0.3$ & $1.9 \pm 0.2$ & $<0.41$ & 19.7 & 5.8 & 3.13 & -67.2 \\
\hline & & 43.4 & $1.4 \pm 0.3$ & $1.3 \pm 0.2$ & $<0.27$ & 23.6 & 2.89 & 1.6 & -67.3 \\
\hline & & 86.8 & $1.0 \pm 0.2$ & $1.0 \pm 0.2$ & $<0.15$ & 22.8 & 1.43 & 0.78 & -70.7 \\
\hline 2014-09-27 & 56928 & 21.7 & $1.8 \pm 0.2$ & $1.8 \pm 0.1$ & $0.83 \pm 0.06$ & 10.2 & 5.29 & 3.25 & -88.5 \\
\hline \multirow[t]{3}{*}{$2014-10-29$} & 56959 & 21.7 & $1.9 \pm 0.2$ & $1.8 \pm 0.2$ & $1.08 \pm 0.09$ & 12.2 & 5.43 & 3.16 & 89.9 \\
\hline & & 43.4 & $1.4 \pm 0.1$ & $1.3 \pm 0.1$ & $0.55 \pm 0.04$ & 6.7 & 2.72 & 1.59 & 89.7 \\
\hline & & 86.8 & $1.1 \pm 0.2$ & $1.0 \pm 0.1$ & $0.32 \pm 0.04$ & 15.5 & 1.45 & 0.78 & 84.7 \\
\hline
\end{tabular}

Notes. The columns show (1) the mean observing epoch, (2) the corresponding MJD, (3) the central observing frequency, (4) the core flux density, (5) the peak intensity, (6) the core FWHM size, (7) the image rms noise level after subtracting the Gaussian model, (8) and (9) the major and minor axis of the elliptical observing beam, and (10) the beam position angle in degree (zero to north, increase in $\mathrm{CCW}$ ). 
Table B.1. continued.

\begin{tabular}{|c|c|c|c|c|c|c|c|c|c|}
\hline $\begin{array}{c}\text { Epoch } \\
\text { (yyyy-mm-dd) } \\
(1)\end{array}$ & $\begin{array}{l}\text { MJD } \\
(2) \\
\end{array}$ & $\begin{array}{c}v_{\mathrm{obs}} \\
(\mathrm{GHz}) \\
(3) \\
\end{array}$ & $\begin{array}{l}S_{v} \\
(\mathrm{Jy}) \\
(4) \\
\end{array}$ & $\begin{array}{c}S_{\text {peak }} \\
\left(\mathrm{Jy} \mathrm{beam}^{-1}\right) \\
(5)\end{array}$ & $\begin{array}{c}d \\
(\mathrm{mas}) \\
(6) \\
\end{array}$ & $\begin{array}{c}\sigma_{\mathrm{rms}} \\
\left(\mathrm{mJy} \mathrm{beam}^{-1}\right) \\
(7)\end{array}$ & $\begin{array}{c}B_{\text {maj }} \\
(\mathrm{mas}) \\
(8) \\
\end{array}$ & $\begin{array}{c}B_{\min } \\
(\operatorname{mas}) \\
(9) \\
\end{array}$ & $\begin{array}{c}B_{\mathrm{PA}} \\
(\mathrm{deg}) \\
(10)\end{array}$ \\
\hline \multirow[t]{3}{*}{ 2014-11-28 } & 56990 & 21.7 & $2.0 \pm 0.3$ & $1.8 \pm 0.2$ & $1.55 \pm 0.15$ & 16.4 & 5.24 & 3.24 & -87.8 \\
\hline & & 43.4 & $1.5 \pm 0.2$ & $1.4 \pm 0.1$ & $0.55 \pm 0.04$ & 9.2 & 2.64 & 1.65 & -79.5 \\
\hline & & 86.8 & $1.5 \pm 0.3$ & $1.2 \pm 0.2$ & $0.44 \pm 0.04$ & 13.6 & 1.38 & 0.81 & 87.2 \\
\hline \multirow[t]{2}{*}{ 2014-12-26 } & 57017 & 21.7 & $1.9 \pm 0.2$ & $1.8 \pm 0.2$ & $<0.34$ & 13.9 & 5.57 & 3.1 & -76.1 \\
\hline & & 86.8 & $1.1 \pm 0.4$ & $0.8 \pm 0.2$ & $0.50 \pm 0.13$ & 49.4 & 1.41 & 0.77 & -74.7 \\
\hline \multirow[t]{3}{*}{ 2015-01-15 } & 57037 & 21.7 & $1.8 \pm 0.2$ & $1.7 \pm 0.1$ & $1.3 \pm 0.1$ & 10.8 & 5.56 & 3.16 & 86.8 \\
\hline & & 43.4 & $1.4 \pm 0.1$ & $1.4 \pm 0.1$ & $0.52 \pm 0.03$ & 5.4 & 2.66 & 1.68 & 88.7 \\
\hline & & 86.8 & $1.3 \pm 0.3$ & $1.1 \pm 0.2$ & $0.43 \pm 0.06$ & 23.1 & 1.41 & 0.79 & -87.1 \\
\hline \multirow[t]{4}{*}{ 2015-03-26 } & 57108 & 21.7 & $2.1 \pm 0.2$ & $2.0 \pm 0.2$ & $0.54 \pm 0.04$ & 12.8 & 5.34 & 3.25 & -85.5 \\
\hline & & 43.4 & $1.5 \pm 0.2$ & $1.4 \pm 0.1$ & $0.45 \pm 0.04$ & 13.1 & 2.61 & 1.67 & -84.8 \\
\hline & & 86.8 & $1.3 \pm 0.2$ & $1.3 \pm 0.2$ & $0.41 \pm 0.05$ & 9.6 & 1.36 & 0.79 & -85.0 \\
\hline & & 129.3 & $0.9 \pm 0.3$ & $0.8 \pm 0.3$ & $<0.07$ & 9.4 & 0.89 & 0.55 & -82.4 \\
\hline \multirow[t]{3}{*}{ 2015-04-30 } & 57142 & 21.7 & $2.0 \pm 0.3$ & $2.0 \pm 0.2$ & $<0.35$ & 15.9 & 5.49 & 3.12 & 89.0 \\
\hline & & 43.4 & $1.2 \pm 0.1$ & $1.3 \pm 0.1$ & $<0.12$ & 5.0 & 2.74 & 1.56 & 87.1 \\
\hline & & 86.8 & $1.1 \pm 0.2$ & $1.0 \pm 0.1$ & $0.17 \pm 0.02$ & 19.6 & 1.44 & 0.75 & 88.7 \\
\hline \multirow[t]{3}{*}{$2015-10-23$} & 57318 & 21.7 & $1.8 \pm 0.3$ & $1.7 \pm 0.2$ & $<0.4$ & 17.7 & 5.79 & 3.07 & -87.0 \\
\hline & & 43.4 & $1.5 \pm 0.2$ & $1.4 \pm 0.1$ & $0.59 \pm 0.04$ & 7.5 & 2.78 & 1.59 & 86.7 \\
\hline & & 86.8 & $1.2 \pm 0.2$ & $1.0 \pm 0.2$ & $0.37 \pm 0.05$ & 21.7 & 1.5 & 0.76 & 81.3 \\
\hline \multirow[t]{3}{*}{ 2015-11-30 } & 57356 & 21.7 & $1.8 \pm 0.2$ & $1.8 \pm 0.2$ & $<0.36$ & 14.2 & 5.54 & 3.24 & 80.6 \\
\hline & & 43.4 & $1.5 \pm 0.2$ & $1.4 \pm 0.1$ & $<0.17$ & 10.0 & 2.73 & 1.64 & 81.5 \\
\hline & & 86.8 & $1.3 \pm 0.2$ & $1.1 \pm 0.1$ & $0.33 \pm 0.03$ & 10.1 & 1.42 & 0.79 & 77.8 \\
\hline \multirow[t]{4}{*}{$2015-12-28$} & 57384 & 21.7 & $2.2 \pm 0.3$ & $2.1 \pm 0.2$ & $<0.38$ & 20.2 & 5.33 & 3.26 & -89.5 \\
\hline & & 43.4 & $1.9 \pm 0.2$ & $1.8 \pm 0.1$ & $0.53 \pm 0.03$ & 7.5 & 2.64 & 1.65 & -88.5 \\
\hline & & 86.8 & $1.5 \pm 0.2$ & $1.3 \pm 0.1$ & $0.39 \pm 0.04$ & 11.7 & 1.37 & 0.79 & -89.7 \\
\hline & & 129.3 & $0.7 \pm 0.2$ & $0.7 \pm 0.2$ & $0.18 \pm 0.05$ & 10.8 & 0.9 & 0.54 & -88.3 \\
\hline \multirow[t]{4}{*}{ 2016-01-13 } & 57401 & 21.7 & $2.2 \pm 0.2$ & $2.2 \pm 0.2$ & $0.77 \pm 0.05$ & 10.2 & 5.72 & 3.19 & 79.1 \\
\hline & & 43.4 & $2.0 \pm 0.3$ & $1.7 \pm 0.2$ & $0.80 \pm 0.09$ & 19.5 & 2.87 & 1.59 & 80.5 \\
\hline & & 86.8 & $1.5 \pm 0.2$ & $1.2 \pm 0.1$ & $0.45 \pm 0.04$ & 11.2 & 1.48 & 0.77 & 79.8 \\
\hline & & 129.3 & $0.8 \pm 0.3$ & $0.8 \pm 0.2$ & $<0.1$ & 16.8 & 0.98 & 0.53 & 80.7 \\
\hline \multirow[t]{4}{*}{ 2016-03-01 } & 57448 & 21.7 & $2.0 \pm 0.3$ & $2.0 \pm 0.2$ & $<0.35$ & 15.2 & 5.7 & 3.25 & 79.6 \\
\hline & & 43.4 & $1.7 \pm 0.2$ & $1.5 \pm 0.1$ & $0.65 \pm 0.05$ & 9.5 & 2.81 & 1.65 & 82.6 \\
\hline & & 86.8 & $1.1 \pm 0.2$ & $1.0 \pm 0.1$ & $0.32 \pm 0.04$ & 16.9 & 1.44 & 0.8 & 80.6 \\
\hline & & 129.3 & $0.8 \pm 0.2$ & $0.8 \pm 0.2$ & $<0.1$ & 16.6 & 0.96 & 0.55 & 87.0 \\
\hline \multirow[t]{3}{*}{ 2016-04-24 } & 57502 & 21.7 & $2.0 \pm 0.2$ & $1.9 \pm 0.1$ & $<0.3$ & 10.0 & 6.27 & 3.18 & 73.6 \\
\hline & & 43.4 & $1.6 \pm 0.2$ & $1.4 \pm 0.1$ & $0.70 \pm 0.06$ & 8.5 & 3.15 & 1.58 & 73.8 \\
\hline & & 86.8 & $1.0 \pm 0.2$ & $1.0 \pm 0.2$ & $<0.15$ & 21.1 & 1.62 & 0.76 & 73.1 \\
\hline \multirow[t]{3}{*}{ 2016-10-18 } & 57680 & 21.7 & $2.0 \pm 0.2$ & $2.0 \pm 0.2$ & $<0.35$ & 14.6 & 5.6 & 3.24 & 78.9 \\
\hline & & 43.4 & $1.6 \pm 0.2$ & $1.5 \pm 0.1$ & $0.65 \pm 0.05$ & 9.8 & 2.77 & 1.63 & 78.2 \\
\hline & & 86.8 & $1.2 \pm 0.3$ & $1.1 \pm 0.2$ & $<0.18$ & 37.6 & 1.35 & 0.83 & 79.8 \\
\hline \multirow[t]{3}{*}{ 2016-11-27 } & 57719 & 21.7 & $2.2 \pm 0.3$ & $2.1 \pm 0.2$ & $<0.41$ & 22.6 & 5.2 & 3.39 & 88.4 \\
\hline & & 43.4 & $1.3 \pm 0.4$ & $1.3 \pm 0.3$ & $<0.53$ & 68.2 & 3.67 & 1.63 & 67.9 \\
\hline & & 86.8 & $1.2 \pm 0.3$ & $1.2 \pm 0.2$ & $<0.22$ & 40.9 & 1.87 & 0.84 & 64.9 \\
\hline \multirow[t]{3}{*}{ 2016-12-28 } & 57750 & 21.7 & $2.0 \pm 0.2$ & $1.9 \pm 0.2$ & $<0.32$ & 11.8 & 5.6 & 3.25 & 76.9 \\
\hline & & 43.4 & $1.6 \pm 0.2$ & $1.5 \pm 0.1$ & $0.67 \pm 0.05$ & 7.1 & 2.83 & 1.63 & 75.4 \\
\hline & & 86.8 & $1.2 \pm 0.2$ & $1.2 \pm 0.2$ & $<0.14$ & 20.3 & 1.42 & 0.82 & 73.2 \\
\hline
\end{tabular}

